\title{
XUV-laser induced ablation of PMMA with nano-, pico-, and femtosecond pulses
}

\author{
L. Juha ${ }^{a}{ }^{*}$, M. Bittner ${ }^{\text {a, b}}$, D. Chvostova ${ }^{a}$, V. Letal ${ }^{\text {d }}$, J. Krasa ${ }^{\text {a }}$, Z. Otcenasek ${ }^{\text {a, d }}{ }^{\text {, }}$ \\ M. Kozlova a , J. Polan ${ }^{\text {a }}$, A.R. Präg ${ }^{\text {a }}$, B. Rus ${ }^{\text {a }}$, M. Stupka ${ }^{\text {a }}$, J. Krzywinski ${ }^{\text {c, e }}$, \\ A. Andrejczuk ${ }^{\text {c, f }}$, J.B. Pelka ${ }^{\text {c, e }}$, R. Sobierajski ${ }^{g}$, L. Ryc $^{\text {h }}$, J. Feldhaus ${ }^{\text {c }}$, F.P. Boody ${ }^{\mathrm{i}}$, \\ M.E. Grisham ${ }^{\mathrm{j}}$, G.O. Vaschenko ${ }^{\mathrm{j}}$, C.S. Menoni ${ }^{\mathrm{j}}$, J.J. Rocca $^{\mathrm{j}}$

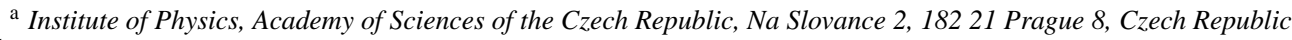 \\ ${ }^{\mathrm{b}}$ Faculty of Mathematics and Physics, Charles University in Prague, Ke Karlovu 3, 12116 Prague 2, Czech Republic \\ ${ }^{\mathrm{c}}$ HASYLAB/DESY, Notkestrasse 85, 22603 Hamburg, Germany \\ ${ }^{\mathrm{d}}$ Faculty of Nuclear Sciences and Engineering Physics, Czech Technical University in Prague, Brehova 7, 11519 Prague 1, Czech Republic \\ ${ }^{\mathrm{e}}$ Institute of Physics, Polish Academy of Sciences, Al. Lotnikow 32/46, PL-02-668 Warsaw, Poland \\ ${ }^{\mathrm{f}}$ Institute of Experimental Physics, University of Bialystok, Lipowa 41, PL-15-424 Bialystok, Poland \\ g Faculty of Physics, Warsaw University of Technology, Ul.Sw A. Boboli 8, PL-02-525 Warsaw, Poland \\ ${ }^{\mathrm{h}}$ Institute of Plasma Physics and Laser Microfusion, Ul. Hery 23, PL-00-908 Warsaw, Poland

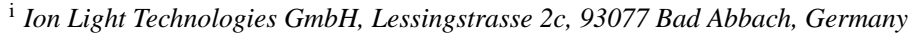 \\ j NSF ERC for Extreme Ultraviolet Science and Technology and Department of Electrical and Computer Engineering, \\ Colorado State University, Fort Collins, CO 80523, USA
}

Available online 24 February 2005

\begin{abstract}
For conventional wavelength (UV-vis-IR) lasers delivering radiation energy to the surface of materials, ablation thresholds, etch (ablation) rates, and the quality of ablated structures often differ dramatically between short (typically nanosecond) and ultrashort (typically femtosecond) pulses. Various very short-wavelength $(\lambda<100 \mathrm{~nm})$ lasers, emitting pulses with durations ranging from $\sim 10$ fs to $\sim 1 \mathrm{~ns}$, have recently been placed into routine operation. This has facilitated the investigation of how ablation characteristics depend on the pulse duration in the XUV spectral region. Ablation of poly(methyl methacrylate) (PMMA) induced by three particular short-wavelength lasers emitting pulses of various durations, is reported in this contribution.
\end{abstract}

(C) 2005 Elsevier B.V. All rights reserved.

Keywords: XUV lasers; Soft X-ray lasers; Ablation; Pulse duration effects; Wavelength effects; Poly(methyl methacrylate); PMMA

\section{Introduction}

Ablation characteristics often differ dramatically with normal wavelength (100's of nm) lasers, depending on whether the radiation energy is delivered to the material surface in either short (nanosecond) or ultrashort (femtosecond) pulses $[1,2]$. In this contribution, we present the first results of our experiments focused on evaluation of pulse duration effects

\footnotetext{
* Corresponding author. Tel.: +420 266052 741; fax: +420286890265.

E-mail address: juha@fzu.cz (L. Juha).
}

in material ablation induced by lasers working at wavelengths shorter than $100 \mathrm{~nm}$.

\section{Experimental}

Among numerous XUV lasers available for application experiments (for a review see [3-6]), we used the three systems described below in our ablation experiments.

The $1.2 \mathrm{~ns}$ pulses of $46.9 \mathrm{~nm}$ radiation from the capillarydischarge Ne-like Ar laser at Colorado State University in Fort Collins, USA [7], were focused by a spherical $\mathrm{Si} / \mathrm{Sc}$ 
multilayer-coated mirror [8] and used for the ablation of organic polymers.

Various materials were irradiated [9-12] with ellipsoidalmirror-focused XUV radiation $(\lambda=86 \mathrm{~nm}, \tau=30-100 \mathrm{fs})$ generated by the free-electron laser (FEL) operated at the TESLA Test Facility (i.e. the TTF1 FEL $[13,14]$ ) in Hamburg, Germany.

The collisionally pumped Ne-like Zn laser $(\lambda=21.2 \mathrm{~nm}$, $\tau<100 \mathrm{ps})[15,16]$ realized at the Prague Asterix Laser System (PALS) Research Center; Prague, Czech Republic was used for irradiating both molecular and elemental solids in the soft X-ray region $(\lambda<30 \mathrm{~nm})$. For the ablation experiments, the soft X-ray laser was focused down to a sub- $100 \mu \mathrm{m}$ spot using a spherical $\mathrm{Mo} / \mathrm{Si}$ multilayer mirror $(60 \mathrm{~cm}$ radius of curvature).

Ablation of PMMA has been extensively investigated with conventional UV-vis-IR lasers. PMMA is also widely used in electron-beam, EUV, and X-ray lithography as a resist so that its radio-chemical and radio-physical properties are well understood. This is why it was chosen for our initial shortwavelength ablation experiments.

Two kinds of PMMA samples were used in our studies. For irradiation at 46.9 and $86 \mathrm{~nm}$, PMMA sheet (1-mm thick; Goodfellow, UK) was fabricated into $2 \mathrm{~mm} \times 5 \mathrm{~mm}$ chips and $13 \mathrm{~mm} \times 32 \mathrm{~mm}$ slabs, respectively. For irradiation at $21.2 \mathrm{~nm}, 500 \mathrm{~nm}$ layers of $495 \mathrm{~K}$ PMMA were deposited on $5 \mathrm{~mm} \times 5 \mathrm{~mm} 315-\mu \mathrm{m}$ thick silicon chips (Silson, UK).

The irradiated surfaces were investigated using profilometry and atomic force microscopy (AFM) performed with an Alpha-Step 500 Surface Profiler (Tencor, USA) and a Dimension 3100 scanning probe microscope (SPM) driven by a NanoScope IV controller (Veeco, USA).

\section{Computer simulation}

The ABLATOR code [17-19] was used to account for the wavelength differences between the lasers. It was developed in the early nineties for numerical simulation of ablation of the materials proposed for first walls of inertial confinement fusion (ICF) reactors, induced by broadband emission $(\sim \mathrm{keV})$ from NOVA-driven hohlraum targets. ABLATOR is a thermo-mechanical one-dimensional hydrodynamic code. It uses a Lagrangian finite-difference approach, which means that matter is divided into zones with fixed mass but variable size, position and velocity. Each time step (see Fig. 1), the code computes the energy deposited in each zone by radiation, the heat conducted from the immediately adjacent zones, and the energy due to stress in the material.

The Mie-Grüneisen equation of state (EOS) provides a new pressure that is linear with zone internal energy and dependent on the zone density. Normal stress, given as the difference between the new pressure and the new deviatoric stress, controls further movement of a zone. This is done by

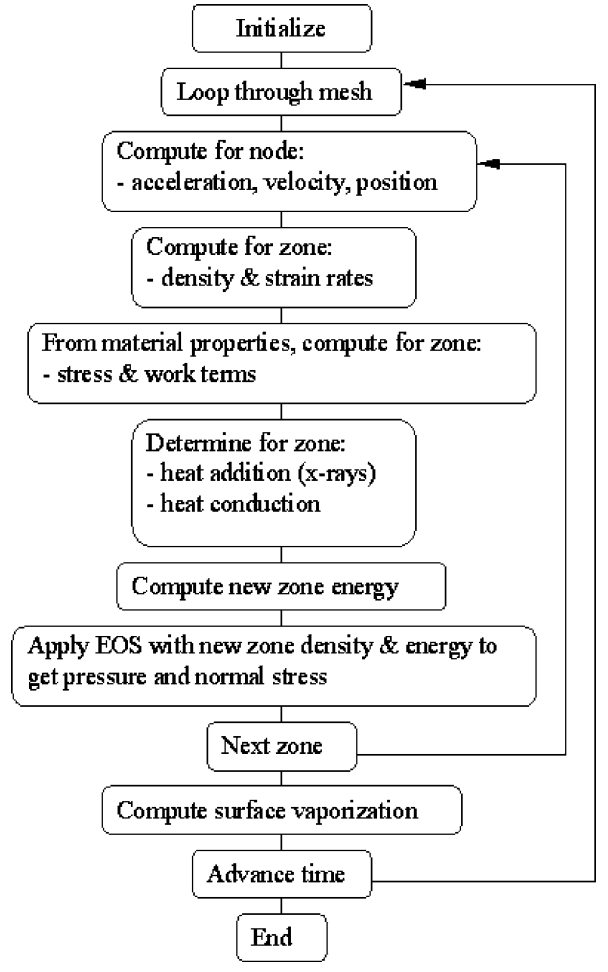

Fig. 1. Flow chart of ABLATOR [17].

calculating zone node movements from the hydrodynamic equation of motion, using normal stress in the zone and its neighbors. When the variables in all zones are actualized, a new cycle starts. In every cycle one zone is designated as the surface, for which the fraction of liquid and vapor is computed, assuming that only surface evaporation occurs. If the vapor/liquid fraction exceeds one, the next zone is designated as the surface. Cracking occurs if the tensile stress exceeds the critical value. For our simulations, the original ABLATOR was modified to model material ablation induced by XUV radiation, which has a much shorter attenuation length than X-rays. The modified code was renamed XUVABLATOR. Efficient absorption of XUV radiation results in a dramatic increase of temperature in the near-surface region of irradiated material, leading to difficulties with the calculation of thermal conduction, very high gradients decreasing simulation stability, etc. Use of the deviatoric stress was also reconsidered. XUV-ABLATOR also takes into account radiation-chemical processes. The most abundant degradation products of PMMA irradiated by a $25 \mathrm{eV}$ electron beam are methylmethacrylate (MMA) and carbon dioxide $\left(\mathrm{CO}_{2}\right)$ [20]. Therefore, the PMMA "vapors" in XUV-ABLATOR are composed of either MMA or $\mathrm{CO}_{2}$. The modified version of the code has been tested by comparing the calculated etch rates for nanosecond pulses of $46.9 \mathrm{~nm}$ radiation to measured values. Calculated values are in good agreement with those obtained with the capillary discharge XUV laser (see Fig. 2). 


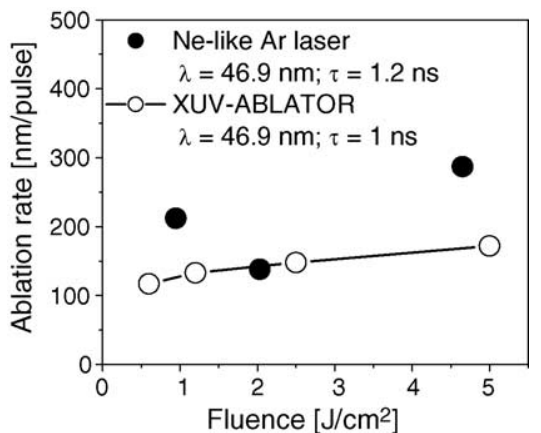

Fig. 2. Calculated and measured fluence dependence of PMMA etch rates for nanosecond pulses of $46.9 \mathrm{~nm}$ radiation.

\section{Results and discussion}

PMMA was cleanly ablated with all three XUV lasers over a wide range of fluences. Hence, etch rates for three different pulse durations have been determined.

The etch rates were further calculated for nanosecond pulses of $86 \mathrm{~nm}$ radiation. By comparing the calculated values for ns pulses with the measured values for sub-100-fs FEL pulses, the influence of pulse duration on XUV ablation efficiency may be evaluated. In Fig. 3, it is shown that sub100 -fs pulses of $86 \mathrm{~nm}$ FEL radiation ablate PMMA at lower rates than predicted by simulation with XUV-ABLATOR for ns pulses at the same wavelength. Taking into account that the ablation occurs in the low-fluence region, where the rate is controlled by attenuation length (for more details see for example $[1,21]$ ) it follows from the results summarized in Fig. 3 that the attenuation length of FEL radiation must be shorter than that derived from the linear absorption coefficient (reported for example in [22] and used as a key input parameter in the XUV-ABLATOR simulation for the nanosecond pulses).

This may be explained if one assumes that absorption is enhanced for ultraintense, ultrashort pulses. In the experiment, the FEL-radiation intensity $\left(>1 \mathrm{TW} / \mathrm{cm}^{2}\right)$ was $10^{4}$ times higher than that considered at the same fluence in the XUV-ABLATOR simulation. Such an enhancement is usually much stronger and a very common phenomenon in the UV-vis-IR spectral regions. Non-linear, multi-photon ab-

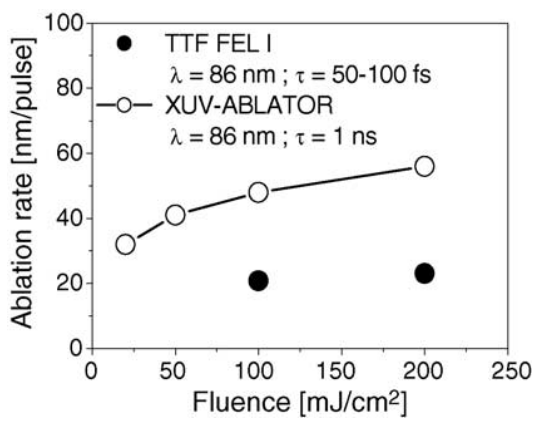

Fig. 3. Calculated and measured PMMA etch rates for nano- and femtosecond pulses of $86 \mathrm{~nm}$ radiation, respectively.

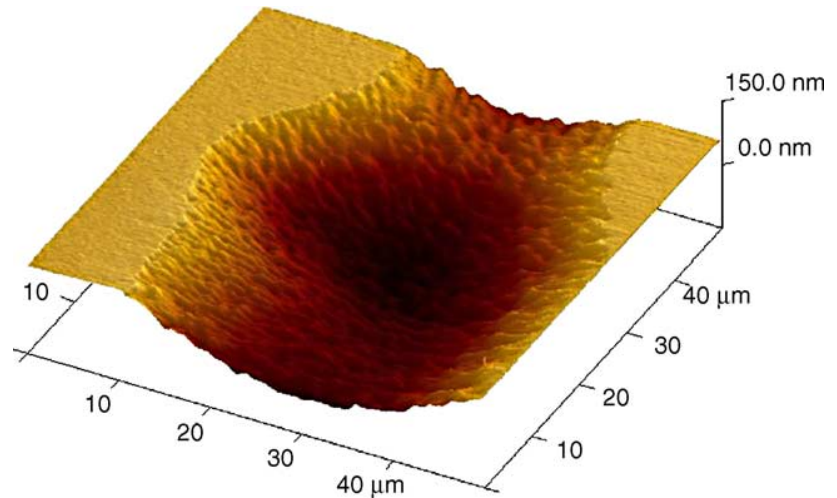

Fig. 4. AFM image (tapping mode) of a crater ablated in PMMA by a single pulse of $21.2 \mathrm{~nm}$ radiation (fluence $\sim 1 \mathrm{~J} / \mathrm{cm}^{2}$; etch rate $150 \mathrm{~nm} /$ pulse).

sorption of eV-photons is responsible for the enhancement in most cases. This effect likely does not play an important role in the XUV spectral region because of the different nature of high-energy-photon interaction with matter. Nevertheless, enhanced absorption of XUV-FEL radiation has been observed in free rare-gas clusters illuminated by the focused radiation delivered from the TTF1 FEL [23]. Its nature is not yet fully understood.

The etch rates determined in the ablation experiment with the sub-100-ps pulses of $21.2 \mathrm{~nm}$ radiation (see Fig. 4) also seem to be reduced (factor of 2-3) with respect to those calculated by XUV-ABLATOR for nanosecond pulses. However, the reason should be different than that discussed above for sub-100-fs pulses of $86 \mathrm{~nm}$ radiation. Attenuation length shortening is unlikely for sub-100-ps pulses (i.e. lower intensity) of $21.2 \mathrm{~nm}$ radiation because it is hard to imagine a strong non-linear interaction of $58.5 \mathrm{eV}$ photons at intensities around $10 \mathrm{GW} / \mathrm{cm}^{2}$.

\section{Conclusions}

Efficient ablation $(\sim 10-100 \mathrm{~nm} /$ pulse $)$ induced by nano-, pico-, and femtosecond XUV lasers, was observed in PMMA in complementary fluence regimes. The ablation efficiency of different lasers emitting pulses of various durations at different wave-lengths can be indirectly compared using the XUV-ABLATOR computer simulation of ablation induced by nanosecond pulses. First results suggest slightly enhanced absorption of ultra-intense XUV-FEL radiation in the organic polymer. The etch rate is slightly reduced also for sub-100-ps pulses of soft-X-ray laser radiation with respect to the values calculated for nanosecond pulses. However, this reduction cannot be explained in the same way as with ultrafast XUV beams because of the higher photon energy and lower intensity in the soft X-ray beam. Surface quality of irradiated and ablated surfaces is very high for both short and ultrashort pulses. Craters with sharp edges, without bubbles and thermal damage, testify to a key role of non-thermal processes in XUV laser ablation. In conclusion, our initial XUV experi- 
ments do not show the dramatic difference between ablation of organic polymers with short and ultrashort pulses that is seen in the UV-Vis-IR.

\section{Acknowledgements}

This work was partially funded by the Czech Ministry of Education within the framework of programs INGO (Grant 1P2004LA235) and National Research Centers (Grant LN00A100), State Committee for Scientific Research of the Republic of Poland (Grant no. 72/E-67/SPB/5.PR UE/DZ 27/2003-2005), and the European Commission (G1MA-CI2002-4017; CEPHEUS). The Colorado State University researchers acknowledge the support of the U.S. Department of Energy, Chemical Sciences, Geosciences, and Biosciences Division of the Office of Basic Energy Sciences, and the partial support of the Engineering Research Centers Program of the National Science Foundation under NSF Award Number EEC-0310717.

\section{References}

[1] D. Bäuerle, Laser Processing and Chemistry, third ed., Springer-Verlag, Berlin, 2000.

[2] S. Küper, M. Stuke, Appl. Phys. Lett. 54 (1989) 4.

[3] J.J. Rocca, Rev. Sci. Instrum. 70 (1999) 3799.

[4] H. Daido, Rep. Prog. Phys. 65 (2002) 1513.

[5] G.J. Tallents, J. Phys. D36 (2003) 259.

[6] E. Plönjes, J. Feldhaus, T. Möller, Phys. World 16 (7) (2003) 33.

[7] J.J. Rocca, J. Filevich, E.C. Hammarsten, E. Jankowska, B. Benware, M.C. Marconi, B. Luther, A. Vinogradov, I. Artiukov, S.
Moon, V. Shlyaptsev, Nucl. Instrum. Meth. Phys. Res. A507 (2003) 515.

[8] B.R. Benware, A. Ozols, J.J. Rocca, I.A. Artioukov, V.V. Kondratenko, A.V. Vinogradov, Opt. Lett. 24 (1999) 1714.

[9] A. Andrejczuk, U. Hahn, M. Jurek, J. Krzywinski, J. Pelka, H. Reniewicz, E.A. Schneidmiller, W. Sobala, R. Sobierajski, M.V. Yurkov, TTF FEL team, HASYLAB Annual Report, Part 1, 2001, 117.

[10] R. Sobierajski, J. Krzywinski, A. Andrejczuk, B. Faatz, F. Felten, S. Jacobi, L. Juha, M. Jurek, A. Kauch, D. Klinger, J.B. Pelka, E. Saldin, E. Schneidmiller, M. Sikora, B. Steeg, M. Yurkov, in: K.J. Kim, S.V. Milton, E. Gluskin (Eds.), Proceeding of 24th International Free Electron Laser Conference \& 9th FEL Users Workshop, Argonne IL, September 9-13, 2002, Elsevier, Amsterdam, 2003, p. II77.

[11] L. Juha, et al., Nucl. Instrum. Meth. Phys. Res. 507 (2003) 577.

[12] B. Steeg, L. Juha, J. Feldhaus, S. Jacobi, R. Sobierajski, C. Michaelsen, A. Andrejczuk, J. Krzywinski, Appl. Phys. Lett. 84 (2004) 657.

[13] V. Ayvazyan, et al., Eur. Phys. J. D20 (2002) 149.

[14] R. Treusch, C. Gerth, T. Lokajczyk, J. Feldhaus, Nucl. Instrum. Meth. Phys. Res. A467-A468 (2001) 30.

[15] B. Rus, T. Mocek, A.R. Präg, M. Kozlová, G. Jamelot, A. Carillon, D. Ros, D. Joyeux, D. Phalippou, Phys. Rev. A66 (2002), 063806-12.

[16] A.R. Präg, T. Mocek, M. Kozlová, B. Rus, K. Rohlena, Eur. Phys. J. D26 (2003) 59.

[17] A.T. Anderson, Ph.D. Thesis, University of California at Berkeley, Berkeley, 1996

[18] A.T. Anderson, A.K. Burnham, M.T. Tobin, P.F. Peterson, Fusion Technol. 30 (1996) 757.

[19] A.T. Anderson, P.F. Peterson, Exp. Heat Transfer 10 (1997) 51.

[20] H. Hiraoka, IBM J. Res. Dev. 21 (1977) 121.

[21] T. Lippert, J.T. Dickinson, Chem. Rev. 103 (2003) 453.

[22] I.E. Ferincz, C. Toth, J.F. Young, J. Vac. Sci. Technol. B14 (1997) 828.

[23] H. Wabnitz, et al., Nature 420 (2002) 482. 